\author{
Hanson Huang \\ Hans U. Mair \\ Explosion Damage Division \\ (Code 460) \\ Naval Surface Warfare Center \\ Indian Head Division \\ Silver Spring, MD 20903-5640
}

\section{Neoclassical Solution of} Transient Interaction of Plane Acoustic Waves with a Spherical Elastic Shell

\begin{abstract}
A detailed solution to the transient interaction of plane acoustic waves with a spherical elastic shell was obtained more than a quarter of a century ago based on the classical separation of variables, series expansion, and Laplace transform techniques. An eightterm summation of the time history series was sufficient for the convergence of the shell deflection and strain, and to a lesser degree, the shell velocity. Since then, the results have been used routinely for validation of solution techniques and computer methods for the evaluation of underwater explosion response of submerged structures. By utilizing modern algorithms and exploiting recent advances of computer capacities and floating point mathematics, sufficient terms of the inverse Laplace transform series solution can now be accurately computed. Together with the application of the Cesàro summation using up to 70 terms of the series, two primary deficiencies of the previous solution are now remedied: meaningful time histories of higher time derivative data such as acceleration and pressure are now generated using a sufficient number of terms in the series; and uniform convergence around the discontinuous step wave front is now obtained, completely eradicating spurious oscillations due to the Gibbs' phenomenon. New results of time histories of response items of interest are presented. (c) 1996 John Wiley \& Sons, Inc.
\end{abstract}

\section{INTRODUCTION}

A detailed solution to the transient interaction of plane acoustic waves with a spherical elastic shell was obtained more than a quarter of a century ago based on the classical separation of variables, series expansion, and Laplace transform techniques (Huang, 1969). The solution for each term of the series is a rational function of the Laplace transform parameter $s$. The inverse Laplace transforms were obtained by the standard heaviside formula. An eight-term summation of the time history series was sufficient for the convergence of the shell deflection and strain, and to a lesser degree, the shell velocity. Since then, the results have been used by many authors for validation of computational models of submerged structures subject to underwater shock. As recent as in the 1990s, there have been many such applications; a partial list is cited here. Swegle and Attaway (1995) compared the shell radial velocity time histories to those obtained by an advanced numerical technique termed smoothed particle hydrodynamics $(\mathrm{SPH})$, a gridless Lagrangian

Received July 17, 1995; Accepted November 2, 1995.

Shock and Vibration, Vol. 3, No. 2, pp. 85-98 (1996)

(C) 1996 by John Wiley \& Sons, Inc.

CCC 1070-9622/96/02085-14 
technique, to determine the feasibility of using the SPH technique for the analysis of underwater explosion problems involving fluid/structure and shock/structure interactions. For the same purpose, Chisum and Shin (1995) compared the shell radial velocity time histories to those computed by the DYTRAN coupled Eulerian/Lagrangian hydrocode. Hibbit, Karlsson \& Sorensen, Inc. (1992) compared the shell radial velocity time histories to demonstrate the capabilities of the doubly asymptotic approximation (DAA) implementation in the USA-ABAQUS code. Whang (1991) proposed that the results be used as benchmarks for the validation of underwater explosion response computer codes. Chan and Atkatsh (1991) used the deflection, strain, and velocity time histories to test the DAA implementation in the EPSA code. Chan $(1990,1993)$ used the shell radial velocity time histories to demonstrate the validity of his modified finite element (MFE) procedures for underwater shock analysis. Wright (1991) comparerd the shell radial velocity time histories validating his direct time integration methods for structural acoustics. Waldo (1994) also used these time histories to test his virtualsource model for fluid/structure interaction. In all cases, the agreement in the comparison of results is less than perfect. The eight-term solution, nevertheless, was sufficient for these validations. Zhang and Geers (1993) solved this canonical problem again using a different method, and based on which Geers and Ju (1994) developed a computer program to generate results for verifying the correctness and accuracy of FE/boundary-element codes used to analyze the response of marine structures to underwater explosions.

There were two primary deficiencies in the eight-term series solution, namely, meaningful time histories of higher time derivative items such as acceleration and pressure were not generated due to the insufficient number of terms in the series, and uniform convergence around the discontinuous step wave front was not obtained because of the Gibbs' phenomenon. Actually, the asymptotic solution, exact within shell theory for all shell response items of the neighborhood of the first point of impact at very early time (roughly one twentieth of the time for the incident wave front to traverse the diameter of the shell), was previously found by Milenkovic and Raynor (1966) using the approach of geometric acoustics, and by Tang and Yen (1970) using Watson's transformation of the modal series together with the steepest descent method. Because these results are for very early time, they are not suitable, and therefore seldom used, for the validation of shell response results obtainable by other methods. Zhang and Geers (1993) innovatively developed and incorporated a simpler version of this type of early time solution into the modal series and successfully solved the convergence problem for this early space-time domain. The present article addresses these two deficiencies for the entire space-time domain and they have now been remedied with the advent and availability of more powerful computers and increased sophistication of computational algorithms. In particular, a large number of terms (up to 70) of the series solution are now accurately computed, and the Cesàro summation of the series is employed to effectively eradicate all spurious oscillations due to the Gibbs' phenomenon. The series solution now converges uniformly toward the true solution. Thus, the early time waveform of the velocity time history and the time of its maximum can be accurately defined, and meaningful time histories for higher time derivatives (e.g., shell acceleration and pressure acting on the shell) can be obtained. The present work reports such an endeavor and presents refined numerical results. These results are particularly useful for validation of computational models that attempt to reproduce the higher time derivatives, which are significantly more difficult to obtain.

\section{RECAPITULATION OF CLASSICAL SOLUTION}

For the convenience of discussion, the geometry of the problem is sketched in Fig. 1. The origin $\mathrm{O}$ of a Cartesian coordinate system $(x, y, z)$ and a spherical coordinate system $(r, \theta, \phi)$ coincides with the center of the spherical elastic shell. For consistency, the same symbols and notations to denote various quantities in the original article (Huang, 1969) will also be used here. The incident plane wave travels in the negative $z$ direction. The spherical shell is considered to be made of isotropic elastic material and its properties are middle surface radius $a$, thickness $2 h$ ( $h$ is the halfthickness), elastic (Young's) modulus $E$, Poisson ratio $\nu$, and mass density $\rho_{s}$. The middle surface deflections of the shell in the $r$ and $\theta$ directions are denoted by $\delta_{r}$ and $\delta_{\theta}$, respectively. The isotropic and homogeneous fluid medium of infinite extent surrounding the shell is completely characterized by its unperturbed mass density $\rho$ and 

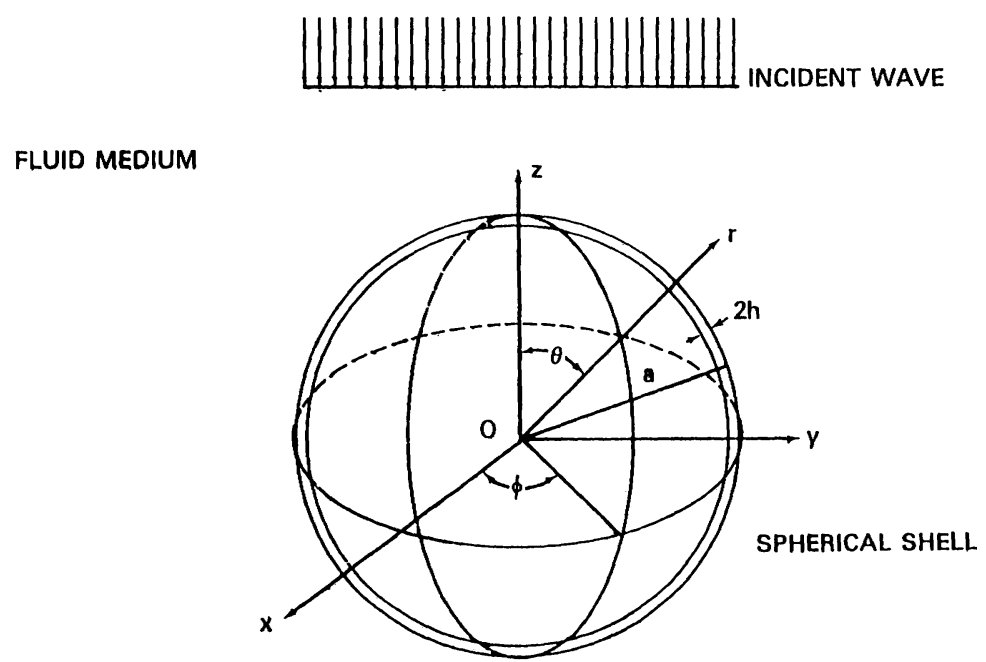

FIGURE 1 Geometry of the problem.

sound speed $c$. The following dimensionless quantities are also used here.

$$
\begin{aligned}
w & =\frac{\delta_{r}}{a}, \quad u=\frac{\delta_{\theta}}{a}, \quad T=\frac{c t}{a}, \quad R=\frac{r}{a}, \quad Z=\frac{z}{a}, \\
C^{2} & =\frac{E}{\rho_{s}(1-\nu) c^{2}}, \quad M=\frac{\rho a}{2 h \rho_{s}}, \quad \Pi=\frac{p}{\rho c^{2}},
\end{aligned}
$$

where $t$ and $p$ denote time and pressure, respectively. The total pressure field in the surrounding fluid medium is the sum of two separable parts, i.e.,

$$
\Pi(R, \theta, T)=\Pi^{\mathrm{inc}}(R, \theta, T)+\Pi^{\mathrm{sra}}(R, \theta, T),
$$

where $\Pi^{\text {inc }}$ denotes the incident pressure and $\Pi^{\text {sra }}$ denotes the sum of the scattered and the radiated pressure. Adjusting the origin of the time coordinate such that the incident wavefront impinges upon the vertex of the shell $(R=1, \theta=0)$ at $T=0$, an arbitrary incident plane wave can be expressed as

$$
\begin{aligned}
\Pi^{\mathrm{inc}}(Z, T)= & \Pi^{\mathrm{inc}}(R, \theta, T) \\
= & F(Z-1+T) H(Z-1+T) \\
= & F(R \cos \theta-1+T) \\
& H(R \cos \theta-1+T),
\end{aligned}
$$

where $H$ is the heaviside step function and $F$ is an arbitrary function of its argument. For a step wave, $F=1$, and for an exponentially decaying wave, $F=\exp [-\beta(Z-1+T)]$ where $\beta$ is the dimensionless decay constant. $\Pi^{\text {sra }}$, its first time derivative, the deflection and velocity of the shell have quiescent initial conditions at $T=0$. The boundary condition of the total pressure field at the fluid-shell interface is

$$
\frac{\partial \Pi}{\partial R}=-\frac{\partial^{2} w}{\partial T^{2}} \quad \text { at } R=1,
$$

and $\Pi^{\text {sra }}$ also satisfies the radiation condition at far field. The total pressure loading acting on the shell is $\Pi(1, \theta, T) . \Pi^{\text {inc }}$ and $\Pi^{\text {sra }}$ satisfy the wave equation separately. The same bending theory shell equation of motion in the original work (Huang, 1969) is again used here. The shell deflections and the pressure field can be expanded in terms of Legendre functions as the following:

$$
\begin{aligned}
w(\theta, T) & =\sum_{m=0}^{\infty} w_{m}(T) P_{m}(\cos \theta), \\
u(\theta, T) & =\sum_{m=1}^{\infty} u_{m}(T) \frac{d}{d \theta} P_{m}(\cos \theta), \\
\Pi(R, \theta, T) & =\sum_{m=0}^{\infty} \Pi_{m}(R, T) P_{m}(\cos \theta) .
\end{aligned}
$$

Applying the Laplace transform with respect to $T$ with $s$ as the transform parameter, solving the wave equation for $\Pi^{\text {sra }}$ and the shell equation of motion for the deflections satisfying the initial and boundary conditions, the Laplace transformed solution of the modal radial deflection of the shell is obtained (Huang, 1969).

$$
\bar{w}_{m}(s)=\frac{-s f(s)(2 m+1) M\left(s^{2}+C_{m}\right) s^{m-1}}{Q_{m}(s)},
$$


where an overbar designates Laplace transformed quantities, $f(s)$ is the Laplace transform of $F(T)$, and

$$
C_{m}=C^{2}\left(1+\frac{h^{2}}{3 a^{2}}\right) \frac{m(m+1)-(1-\nu)}{1+\nu} .
$$

On the right-hand side of Eq. (6), the denominator $Q_{m}$ is a function of the shell and fluid properties. It is a finite polynomial of $(m+5)$ th degree with real and positive coefficients. For the detailed expression of $Q_{m}$ and other quantities, the reader is again referred to the original article (Huang, 1969). The right-hand side of Eq. (6) is therefore a rational function and its inverse Laplace transform can be accurately computed as long as the roots of $Q_{m}$ can be accurately found. Expressions similar to Eq. (6), involving the same $Q_{m}$, can also be written for other quantities of interest such as shell strain and the total pressure.

\section{GIBBS' PHENOMENON AND CESÀRO'S SUMMATION}

The Laplace transform of the expression for the incident pressure, Eq. (3), is

$$
\begin{aligned}
\bar{\Pi}^{\text {inc }}(Z, s) & =f(s) \exp [-(1-Z) s] \\
& =f(s) \exp [-(1-R \cos \theta) s],
\end{aligned}
$$

and its series expansion in terms of Legendre functions is

$$
\begin{aligned}
\bar{\Pi}^{\mathrm{inc}}(R, \theta, s)= & f(s) e^{-s} \sum_{m=0}^{\infty}(2 m+1) \\
& \sqrt{\frac{\pi}{2 R s}} I_{m+1 / 2}(R s) P_{m}(\cos \theta),
\end{aligned}
$$

where $\sqrt{\pi / 2 R S} I_{m+1 / 2}(R s)$ is a modified Bessel function of the first kind. For a unit step wave, $f(s)=1 / s$, and or an exponentially decaying wave, $f(s)=1 /(s+\beta)$. It can be shown that for a step wave at $R=1$, the inverse Laplace transform of the series in Eq. (9) is

$$
\begin{aligned}
\Pi^{\mathrm{inc}}(1, \theta, T)= & 0.5 T+0.5 \sum_{m=1}^{\infty}\left(P_{m-1}(1-T)\right. \\
& \left.-P_{m+1}(1-T)\right) \\
& \cdot P_{m}(\cos \theta) \quad \text { for } 0 \leq T \leq 2 \\
= & 1.0 \text { for } T \geq 2 .
\end{aligned}
$$

Because the wave front of this step wave is a discontinuity, Gibbs' phenomenon appears in the interval $0 \leq T \leq 2$, when Eq. (10) is used to compute the time histories of $\Pi^{\text {inc }}$, such that the series in Eq. (10) never converges to the true wave form of the unit step wave. Because $\Pi^{\text {inc }}$ is the excitation pressure to the shell, it is obvious that Gibbs' phenomenon also appears in the shell response time histories, predominantly the interval $0 \leq T \leq 2$ and will propagate beyond this time interval depending on the damping characteristics of the system. It is known that the use of Cesàro type of summation of the series could eradicate the Gibbs' phenomenon effects. Previous applications of Cesàro summations to this problem (Berger, 1972; Geers and Ju, 1994), using only the first nine terms of the series, did not sufficiently show the effect of the remedy for higher time derivative items such as velocity, acceleration, and pressure. It will be demonstrated here that a sufficiently large number of terms is required in the Cesàro sum of the series in Eq. (10) to approach the true sum. Using the first $N$ terms of the series, the (C1) Cesàro sum can be written as (Whittaker and Watson, 1958)

$$
\begin{aligned}
\Pi^{\text {inc }}(1, \theta, T)= & 0.5 T+0.5 \sum_{m=1}^{N} \\
& {\left[P_{m-1}(1-T)-P_{m+1}(1-T)\right] } \\
& \left(1-\frac{m-1}{N}\right) P_{m}(\cos \theta)
\end{aligned}
$$

for $0 \leq T \leq 2$.

The above Cesàro sum was mathematically proven to converge to the true sum (Whittaker and Watson, 1958). This formula is also referred to as Fejér's arithmetic means (Carslaw, 1930). The essence of this summation is to gradually reduce the contribution of each term as $m$ increases. It is readily seen from Eq. (11) that if $N$ is small the early $m$ terms could be prematurely and wrongly overreduced. Numerical computations of $\Pi^{\mathrm{inc}}(1, \theta, T)$ at $\theta=0,0.5 \pi$, and $\pi$ computed using regular summation, Eq. (10), and Cesàro summation, Eq. (11), are juxtaposed in the left and right columns and compared to the respective true waveforms in Figs. 2, 3, 4, and 5. The true wave forms of this unit step incident wave at these three locations are unit step functions beginning at $T=0,1.0$, and 2.0, respectively. Figure 2 plots the results of using the first nine terms $(N=8)$ of the series. The spurious 

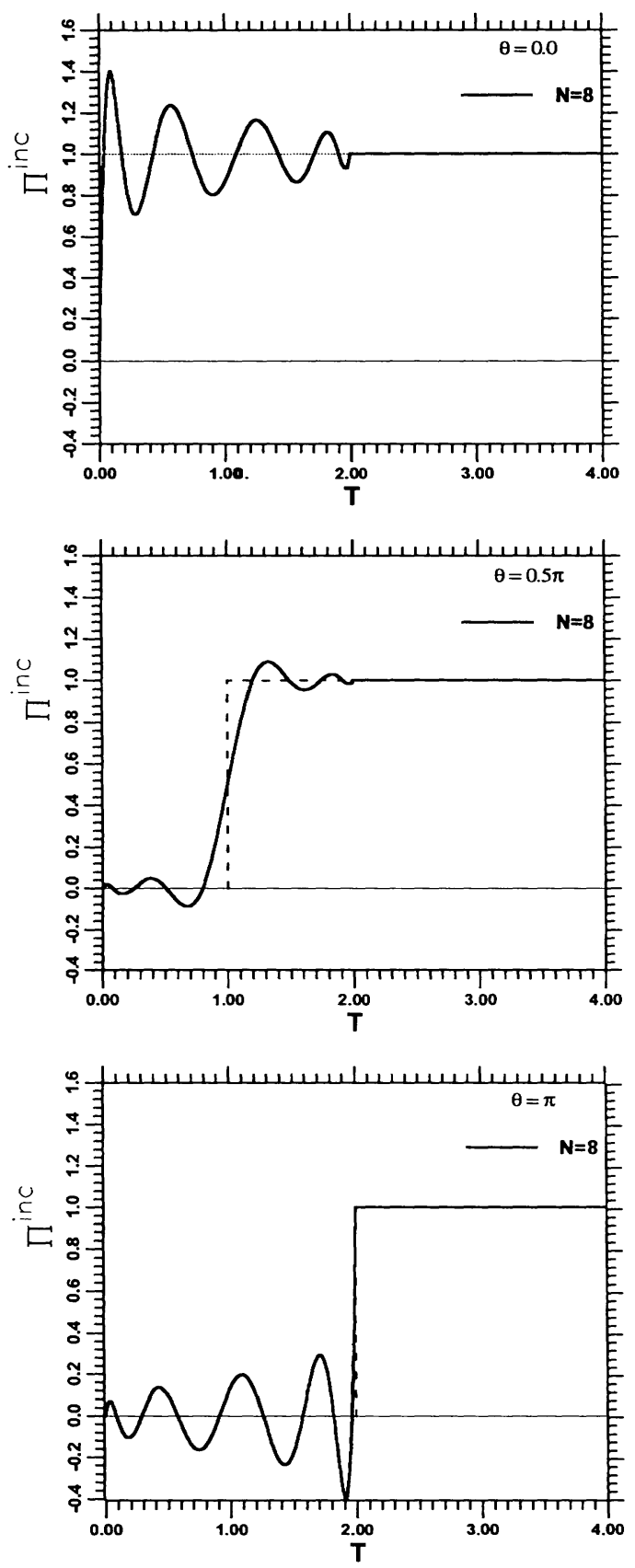
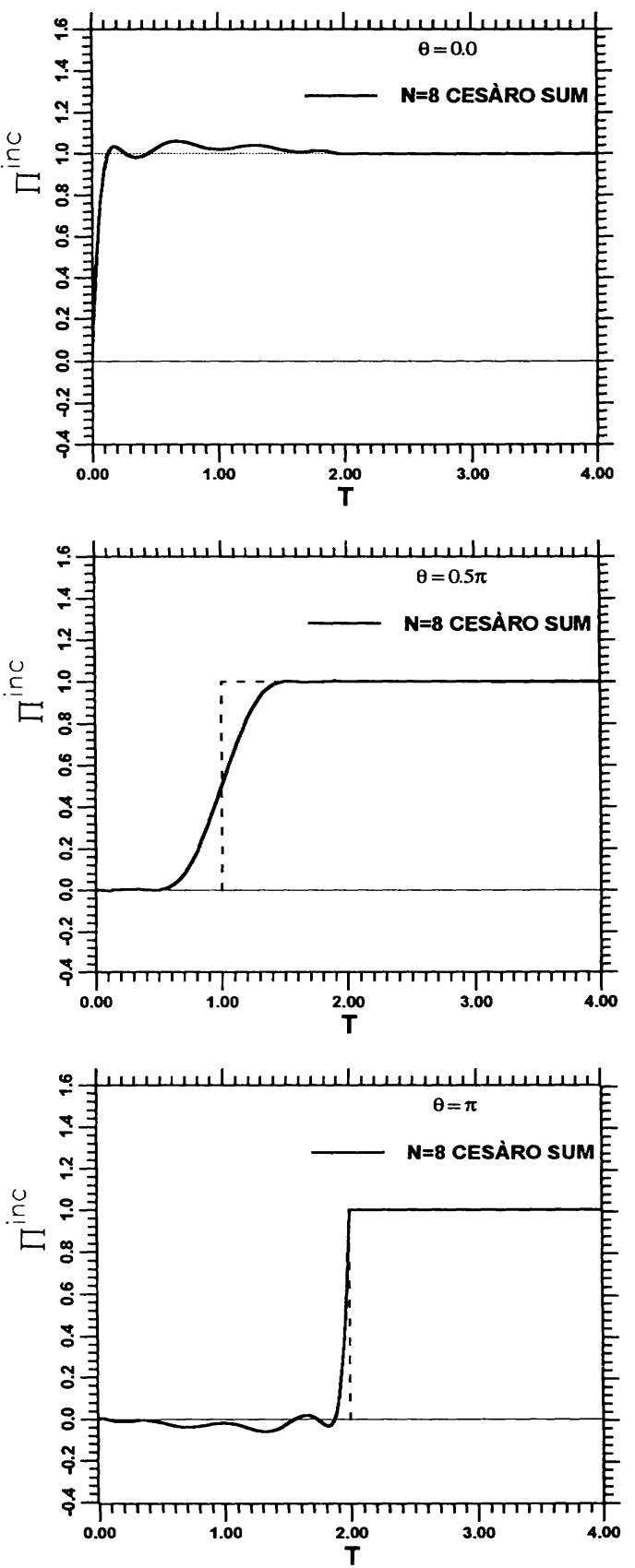

FIGURE 2 Nine-term series representations of $\Pi^{\mathrm{inc}}(1, \theta, T)$.

Gibbs' phenomenon oscillations can be seen from the left column; the amplitude of the oscillations are largest near the wave fronts, and deviate as much as $40 \%$ from the true sum. It can also be seen from the right column that the use of the (C1) Cesàro summation significantly reduces these spurious oscillations but also decreases the steepness of the wave fronts due to the premature reduction of each term of the series as discussed earlier. The use of the first nine terms of the series is far from sufficient to converge to the true waveforms. Figure 3 plots the corresponding results of using the first 51 terms $(N=50)$ of the series. From the left column, it can be seen that the frequency of the Gibbs' phenomenon oscillation increases significantly and the amplitude decreases somewhat. From the right column, it can be seen that the Cesàro summation has basically eradicated all Gibbs' phenomenon oscillation effects and has converged to the true sum except 

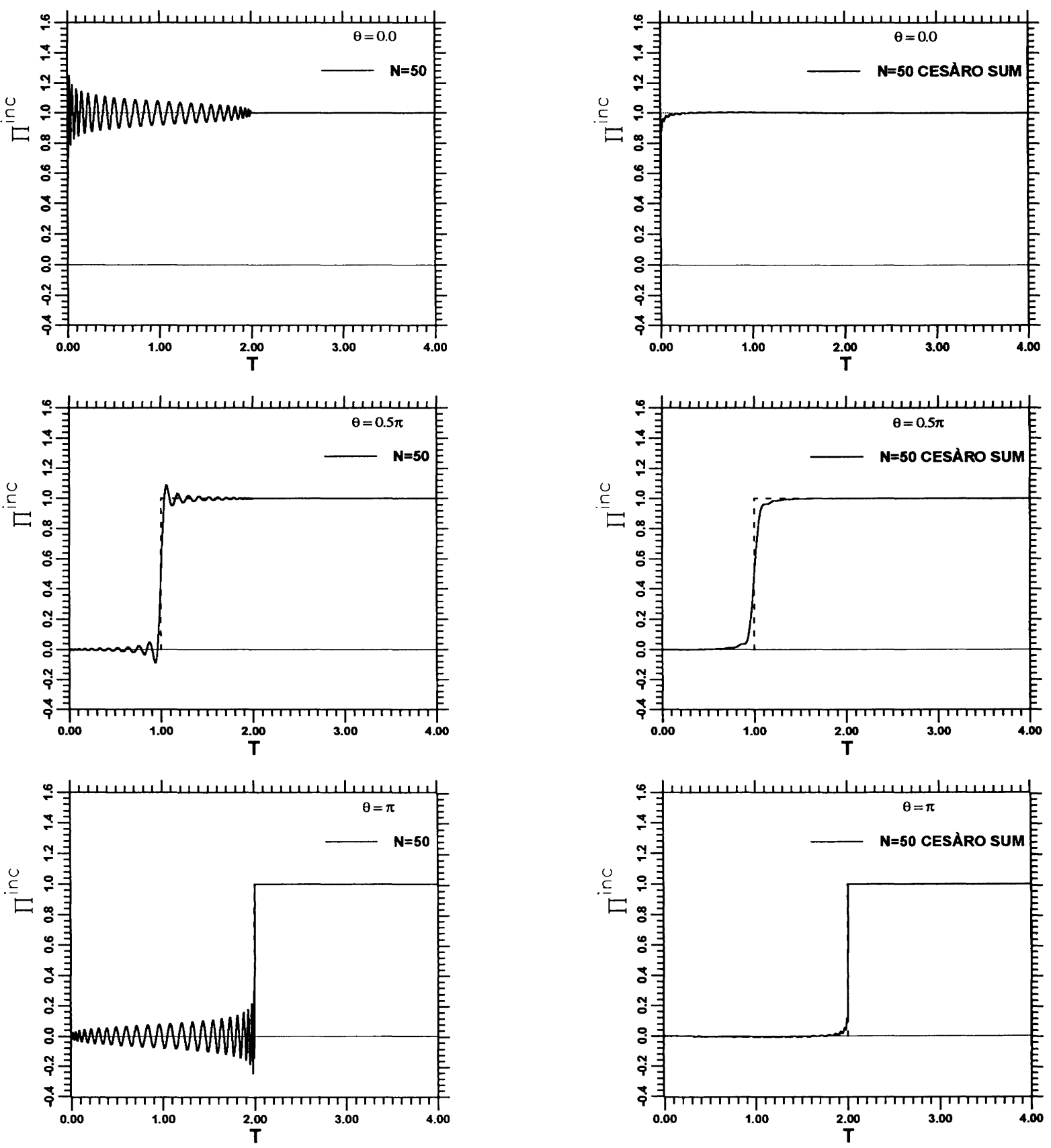

FIGURE 3 Fifty-one-term series representations of $\Pi^{\text {inc }}(1, \theta, T)$. 

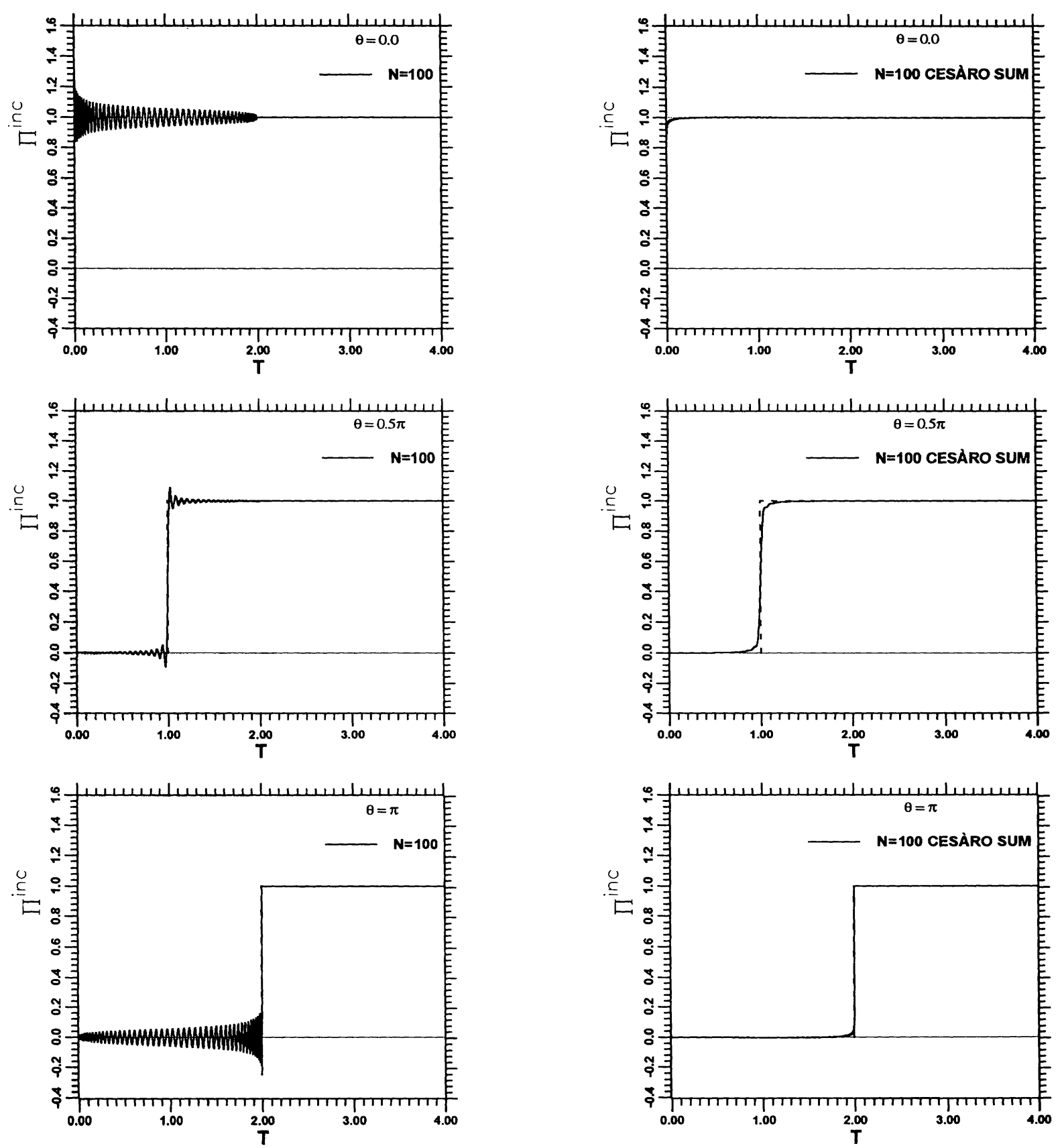

FIGURE 4 One hundred one-term series representations of $\Pi^{\text {inc }}(1, \theta, T)$. 

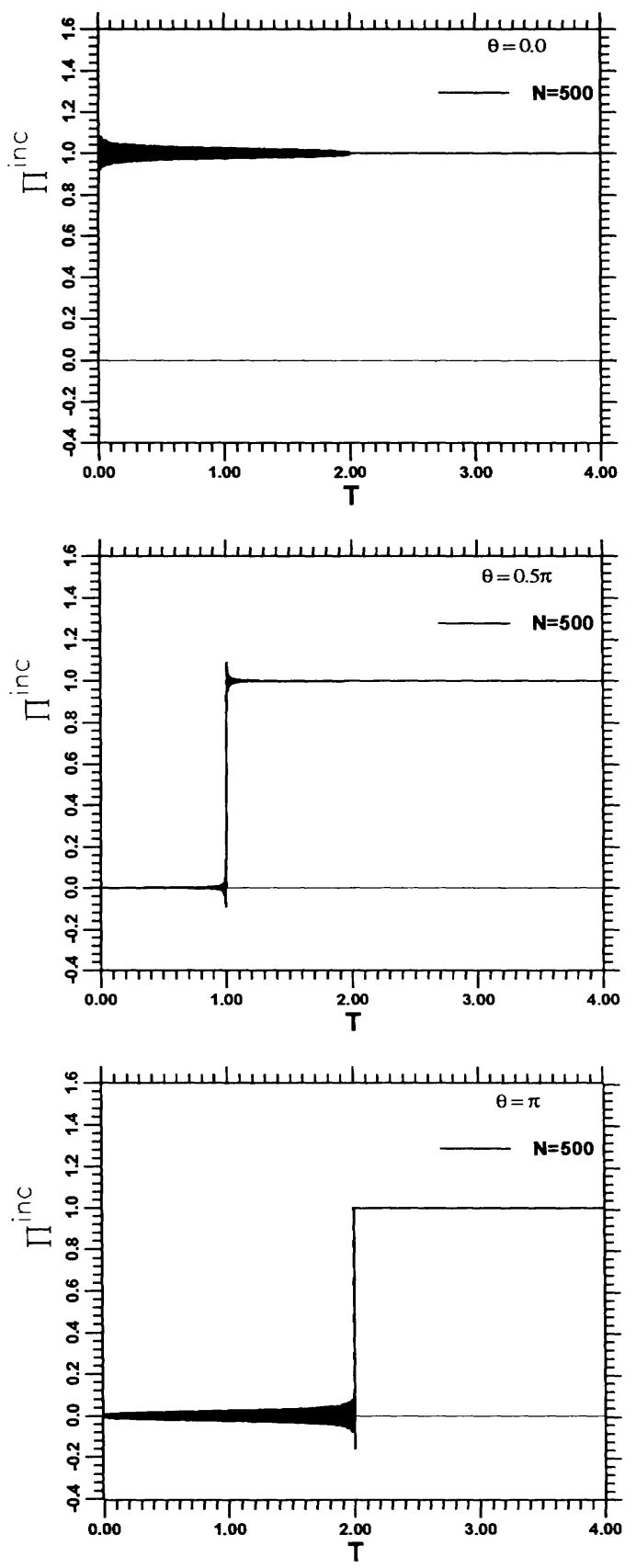
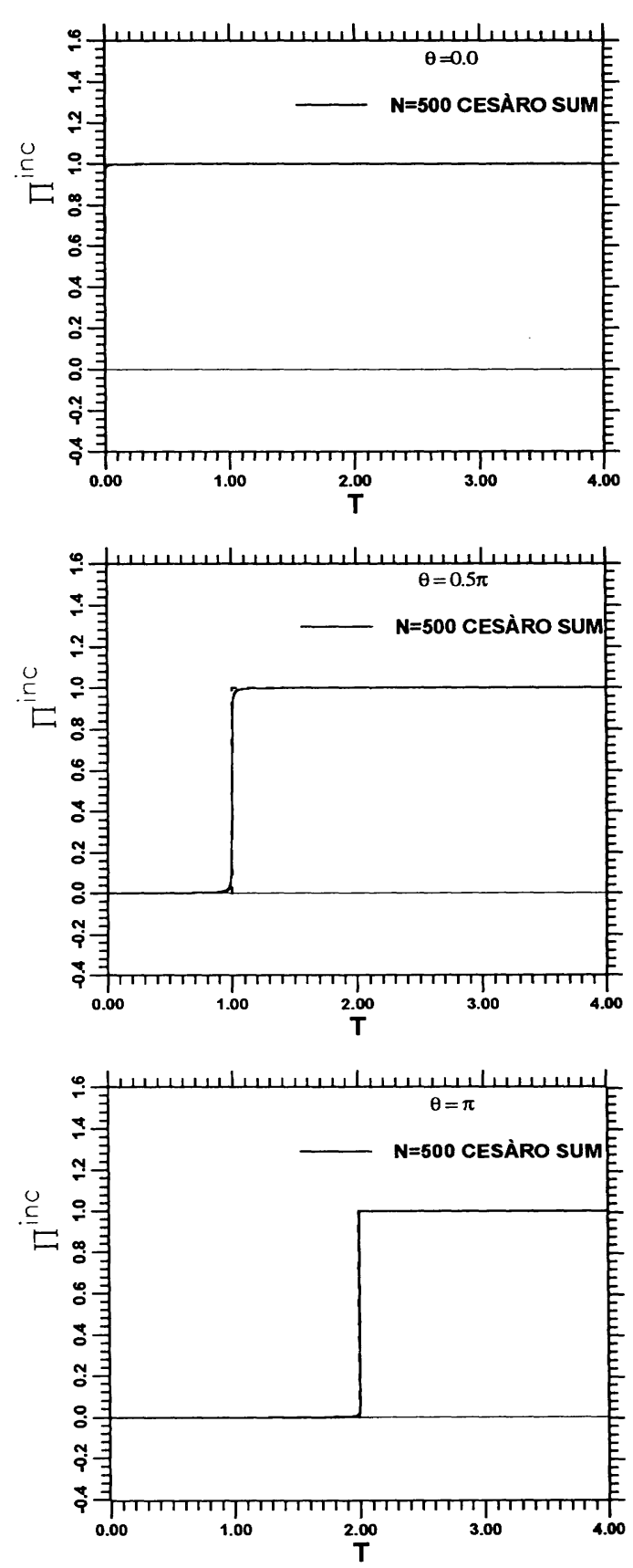

FIGURE 5 Five hundred one-term series representations of $\Pi^{\text {inc }}(1, \theta, T)$.

in small neighborhoods of the wave fronts. The waveforms thus computed could already be acceptable for many applications. Figures 4 and 5 present the results of using $101(N=100)$ and $501(N=500)$ terms of the series, respectively. Again, it is seen from the left columns that with increasing $N$ the amplitudes of the Gibbs' phenomenon oscillations decrease but remain significant near the wave fronts, and that the frequencies of oscillation increase greatly such that the oscillations appear as black bands for the case of $N=500$. From the right columns, it is seen that with increasing $N$, the Cesàro summations further narrow the imperfect neighborhoods of the wave fronts, and for $N=500$ the series representations approach very close to the true waveforms. It is now adequately demonstrated that the series solution to this problem using the $(\mathrm{C} 1)$ Cesàro summation of a sufficiently large number of terms will converge uniformly to the true solu- 
tion. Except in neighborhoods of the wave fronts, the series converges quickly.

\section{NEOCLASSICAL SOLUTION}

To obtain refined time histories of the shell velocity, acceleration, and pressure, large numbers of terms in the modal series are needed. The crux for the inverse Laplace transform of Eq. (6) is the accurate computation of the complex roots of the polynomial $Q_{m}(s)$. The nature of $Q_{m}(s)$ is such that the magnitudes of its coefficients increase rapidly and its roots clutter closely together as $m$ increases; correspondingly, numerical difficulties in floating-point mathematics are encountered. Recently, Jones-Oliveira and Harten (1994) studied the proliferation of roots in this problem. Equation (6) can be simplified by using asymptotic representations of the modified Bessel functions for large $m$, but this is outside the scope of the present article. Here, only the exact $Q_{m}(s)$ was used. Again, the same material properties used in the previous publication (Huang, 1969) for a $2 \%$ (ratio of thickness to radius) steel shell submerged in water were used here: $h / a=0.01, \rho_{s}=486 \mathrm{lb} / \mathrm{ft}^{3}, E=30 \times 10^{6}$ psi, $\nu=0.3, \rho=62.4 \mathrm{lb} / \mathrm{ft}^{3}$, and $c=4794 \mathrm{ft} / \mathrm{s}$. The computations were carried out on personal computers and workstations using a code written in the FORTRAN language. Care was exercised in programming to avoid floating-point pitfalls. Many algorithms for finding the complex roots of a real polynomial were investigated; both Jen- kins' method (Jenkins, 1975) and the eigenvalue method (Press et al., 1992) were employed for cross-checking. Using double precision arithmetic $\left(\mathrm{REAL}{ }^{*} 8\right)$, the complex roots of $Q_{m}(s)$ can be accurately computed up to $m=69$ for the above material properties. Therefore, the inverse Laplace transform of the first 70 terms in the series of Eq. (6) and the corresponding series representations of the shell velocity, acceleration, and pressure were accurately computed. Results for higher $m$ could certainly be obtained using higher precision floating-point arithmetic, but was not pursued here.

The relative radial deflection of the shell between the points $(R=1, \theta=0)$ and $(R=1, \theta=$ $\pi)$ and the radial deflection at $(R=1, \theta=0.5 \pi)$ due to a step incident wave were first recomputed. The present results of regular summations of 8 , 15 , and 30 terms as well as the Cesàro summation of 60 terms of the deflection series all fall on the same curves of Fig. 6. These curves are exactly the same as those of the previously published eight-term sum.

The time histories of shell middle surface hoop strain

$$
\varepsilon_{1}=\frac{1}{a} \frac{\partial \delta_{\theta}}{\partial \theta}+\frac{\delta_{r}}{a}
$$

at $\theta=0,0.5 \pi$, and $\pi$ due a step incident wave were obtained by the Cesàro summation of the first 60 terms in the series, and are plotted in Fig. 7. It can be seen there that due to the focusing effect, the back apex $(\theta=\pi)$ of the shell has the

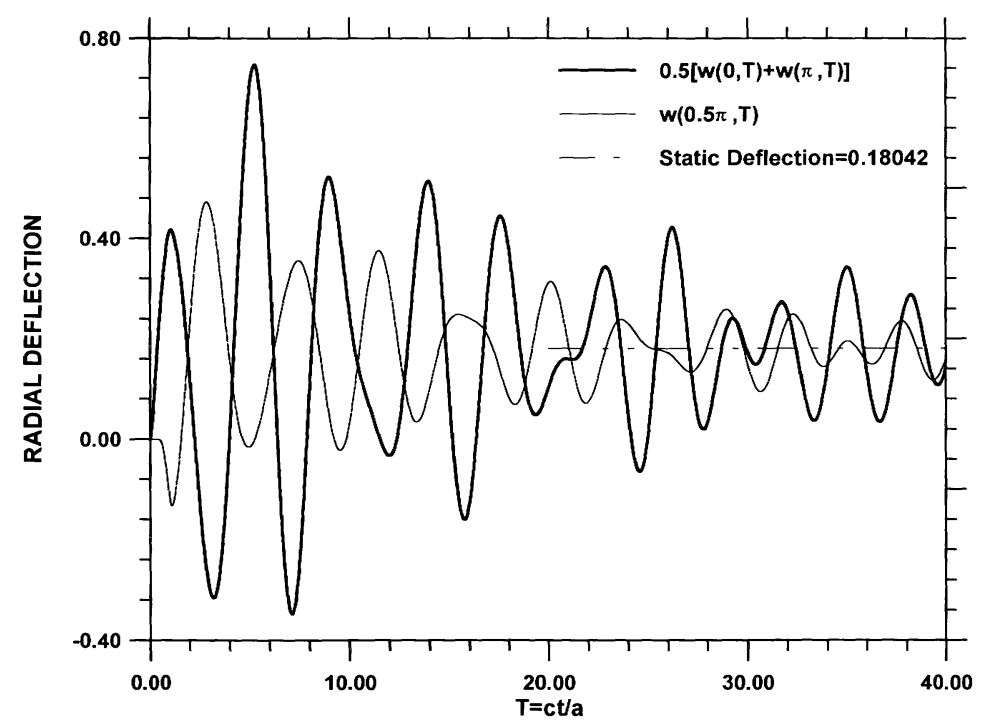

FIGURE 6 Time histories of $w(\theta, T)$ due to a step incident wave. 


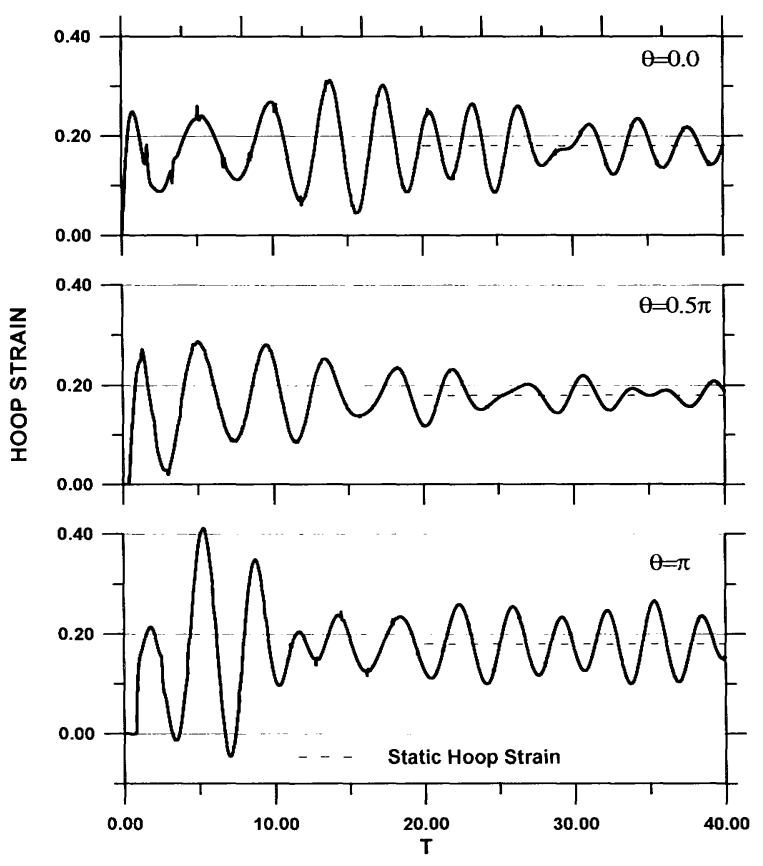

FIGURE 7 Time histories of $\varepsilon_{1}(\theta, T)$ due to a step incident wave.

largest hoop strain. The formula for the arrival time of any disturbance at a given point on the shell derived by Payton (1960) and Tang and Yen (1969) in the present notation is

$$
\begin{aligned}
T_{a}(\theta) & =1-\cos \theta \text { for } \theta \leq \theta_{0}, \\
& =1-\cos \theta_{0}+\left(\theta-\theta_{0}\right) \sin \theta_{0} \quad \text { for } \theta \geq \theta_{0},
\end{aligned}
$$

where

$$
\theta_{0}=\sin ^{-1}\left(\frac{\sqrt{1+\nu}}{C}\right)
$$

Accordingly, the strain and pressure waves arrive at $\theta=0.5 \pi$ and $\pi$ on the shell at $T=0.38784$ and 0.81245 , respectively. The arrival times in Fig. 7 agree closely with these values. Again except for some small spurious oscillations due to the Gibbs' phenomenon, the regular summation of the first eight terms of the series produces the same curves as in Fig. 7. Because the step incident wave is infinitely long, it has masked many details in the strain time histories. Therefore, the hoop strain time histories due to an exponentially decaying incident wave with a decaying time constant equal to one-twentieth of a transit time, i.e., $\beta=10.0$, was also computed using the 60 -term Cesàro sum and is presented in Fig. 8. Here it can be vividly seen that the strain circumnavigates the shell with the shell bending and stretching wave speed as was found in the corresponding cylindrical shell case (Huang, 1970). The time interval between consecutive sharp peaks in the strain time histories of the two apexes of the shell is about 1.7, which is the time required by the strain wave to circumnavigate a distance of $2 \pi$. The corresponding time interval is 0.85 for the $\theta=0.5 \pi$ curve.

The effect of the Gibbs' phenomenon is more pronounced for the regularly summed series for the shell velocity for $0 \leq T \leq 2$. Figure 9 plots the time histories of the shell radial velocity at $\theta=0.0$ due to a step incident wave calculated by the regular summations of the first 9,15 , and 21 terms in the velocity series. Here it can be visualized that the curves oscillate around the true velocity time history in the Gibbs' phenomenon fashion and that near $T=2$ the sums are converging. The same radial velocities calculated by the regular summations of the first 37,39 , and 41 terms in the velocity series is plotted in Fig. 10 where the Gibbs' phenomenon oscillations have smaller amplitudes and higher frequencies but otherwise do not seem to settle in any regular manner. Here the sums converged after $T=1.5$. The bump near $T=1.7$ belongs to the true time history; it is due to the return of the elastic wave initiated at $\theta=0.0$, as can be correlated from Figs. 7 and 8.

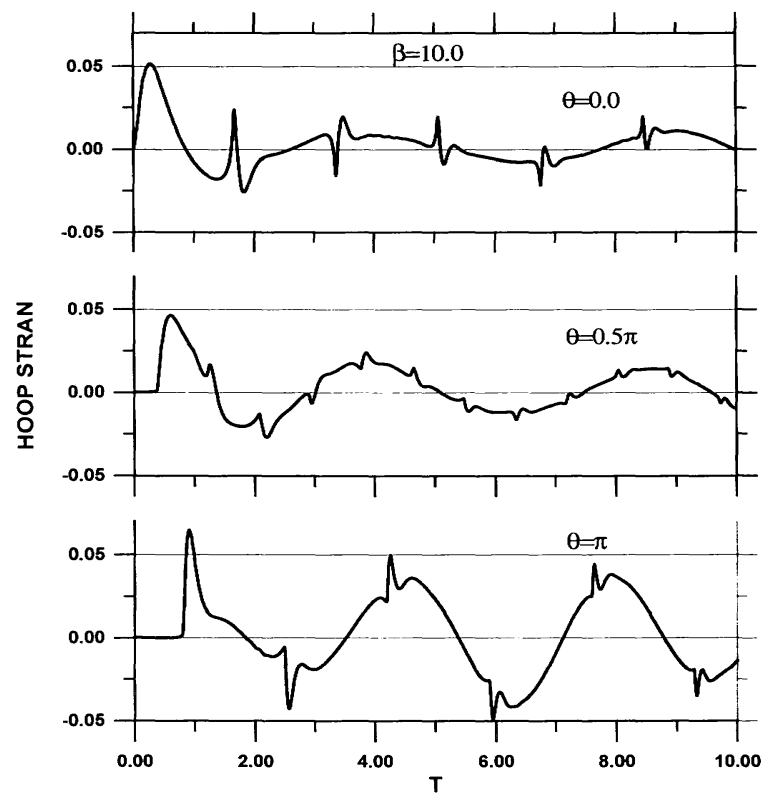

FIGURE 8 Time histories of $\varepsilon_{1}(\theta, T)$ due to a exponentially decaying incident wave. 


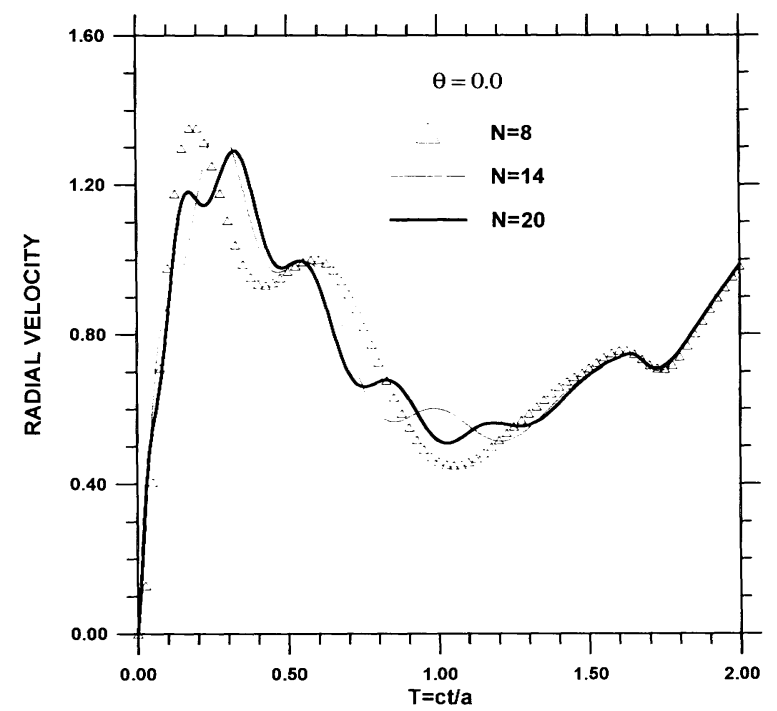

FIGURE 9 Time histories of shell radial velocity due to a step incident wave calculated by the regular summations of 9,15 , and 21 terms of the velocity series.

It is possible to completely eradicate the Gibbs' phenomenon effects and reveal the true time history by the application of the $(\mathrm{C} 1)$ Cesàro summation. Figure 11 shows the time history of the shell radial velocity at $\theta=0.0$ calculated by the Cesàro summation of the first 70 terms of the velocity series, and compares it to that of a nine-term Cesàro sum and that of an asymptotic small time solution obtained by Tang and Yen (1970) using Watson's transformation of the modal series. The result of Tang and Yen (1970) for the shell velocity in the neighborhood of the frontal apex agrees exactly with the earlier solution of Milenkovic and Raynor (1966) using the approach of geometrical acoustics. This asymptotic solution is the true solution for very early time but deviates from the true solution as $T$ increases due to the nature of the mathematical approximations. In Fig. 11, the 70-term Cesàro sum early time velocity and its early time slope (acceleration) agree almost completely with the Watson transformation asymptotic solution. In other words, the "wave front" of the 70-term Cesàro sum velocity very closely approached the true solution. As demonstrated earlier, because the imperfection of the modal series solution using Cesàro summation of a sufficient number of terms is confined to a small neighborhood of the wave front, it can therefore be concluded that the 70-term Cesàro sum here has converged satisfactorily to the true velocity time history. It can also be seen from Fig. 11 that the nine-term Cesàro sum has the correct form

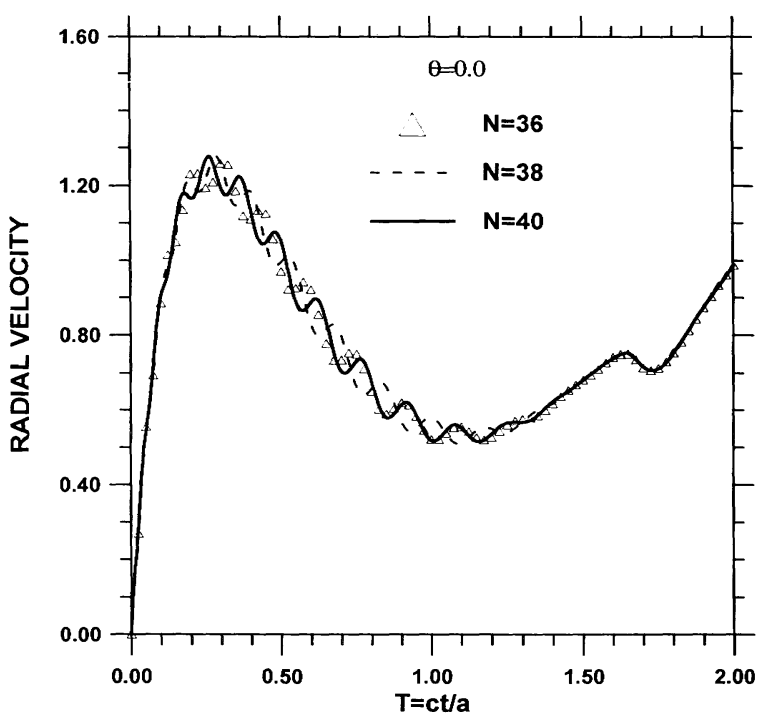

FIGURE 10 Time histories of shell radial velocity due to a step incident wave calculated by the regular summations of 37,39 , and 41 terms of the velocity series.

for the velocity time history, but is insufficient for convergence; the reason for its smaller slope at early time was pointed out in the discussion of Fig. 2. More extensive shell radial velocity histories at $\theta=0.0,0.5 \pi$, and $\pi$ for $0.0 \leq T \leq$ 50.0 using 70-term Cesàro sums are presented in Fig. 12. Here, the very high frequency and very small amplitude oscillations of the velocity curves are due to the terms involving $(h / a)^{2}$ in the shell

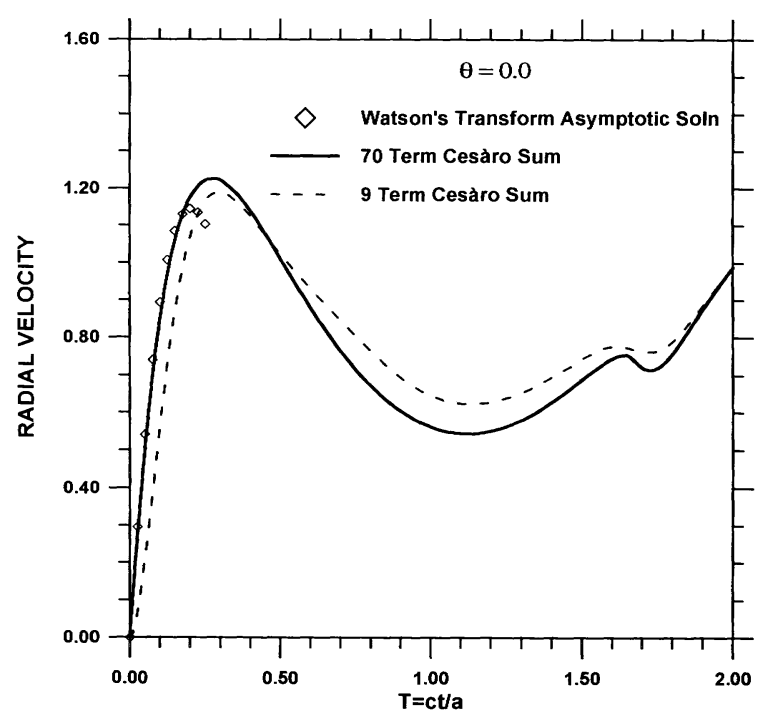

FIGURE 11 Comparison of the Cesàro sum representations of shell radial velocity due to a step incident wave to the Watson's transformation solution. 


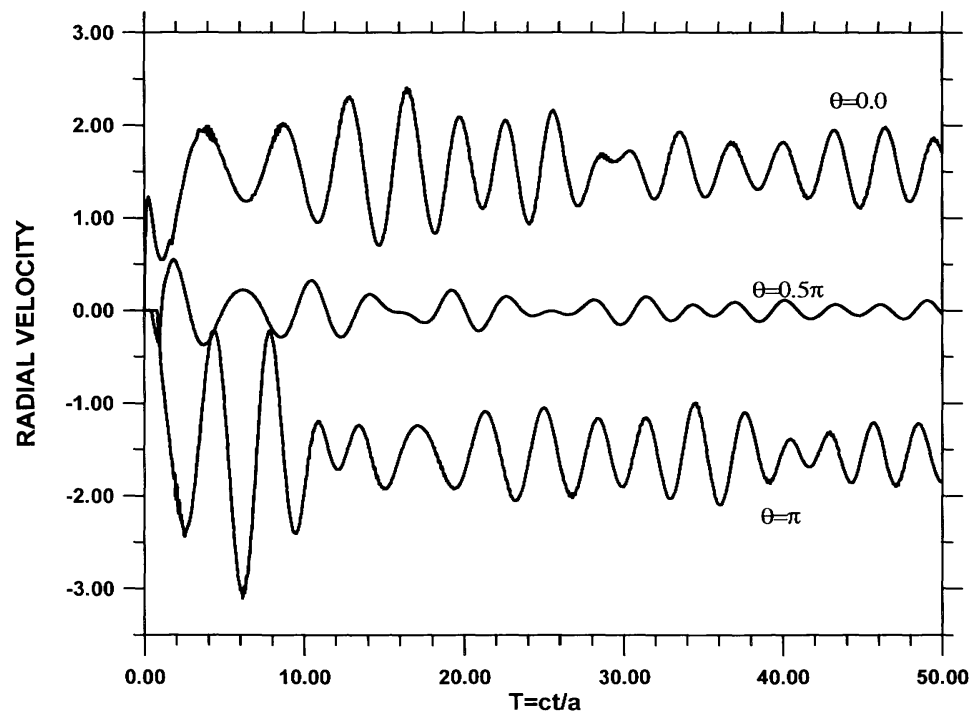

FIGURE 12 Time histories for $0.0 \leq T \leq 50.0$ of shell radial velocity due to a step incident wave.

equations of motion for high $m$ modes (shell theory becomes less valid as $m$ increases). Otherwise, these curves for $T>2.0$ are the same as those previously calculated by the eight-term regular sum (Huang, 1969). The very high $m$ terms in the Legendre series expansions in Eq. (5) only contribute to improving the wave fronts and have no effect outside their immediate neighborhoods. By comparing responses of shells of equal masses made of steel, aluminum, and brass, Milenkovic and Raynor (1966) convincingly demonstrated that near the wave fronts, in a dimensionless time span of 0.1 after the arrival of the disturbance, the inertia effect completely dominates the shell responses. Based on this fact, Zhang and Geers (1993) developed their "partial closed form solution." For the present 70-term series summation, the 70th term $(m=69)$ has a modal structural wavelength equal to about only five shell thicknesses. For the very high $m$ shell modes, it may appear that the use of the present bending shell theory has overstepped its boundary of validity, and that higher order theories such as the one used by Payton (1960) and Tang and Yen (1970) or even 3-dimensional elasticity theory should be used. On the contrary, for the reasons just discussed, it is not necessary to use any better shell theory for the high $m$ shell modes and the use of the present shell theory for the purpose here is still appropriate.

Figure 13 plots the time history of the total pressure acting on the shell at $\theta=0.0$ due to a step incident wave and compares it to the Tang and Yen (1970) Watson's transformation asymptotic solution. The true solution here is that the total pressure reaches the value 2.0 at $T=0.0$ with zero rise time. The 70-term Cesàro sum here starts from 0.0 at $T=0.0$ and reaches a maximum of 1.76459 at $T=0.003$. If the shell has a radius of $5 \mathrm{ft}$, this corresponds to a real rise time of 3 $\mu \mathrm{s}$ and is sufficiently small for most shell damage calculations. The early time decaying slope of the Cesàro sum agrees very well with that of the Watson's transformation solution. The 70-term series solution is not perfect in a small neighborhood of the pressure wave front, as expected, but it fairly closely approached the true solution. After $T=0.04$, the pressure time histories produced by the 40 -term and 50 -term Cesàro sums coincide with that of the 70-term sum. Finally, more extensive time histories of the total pressure acting on the shell at $\theta=0.00,0.5 \pi$, and $\pi$ for $0.0 \leq T \leq 50.0$ due to a step incident wave are presented in Fig. 14. The sharp spike at $T=1.0$ for the $\theta=0.5 \pi$ curve signifies the arrival of the incident wave front; prior to this time the pressure was initiated at $T=0.38784$ by the elastic response of the shell. Due to the imperfection of the calculated wave front here, the magnitude of the spike is lower than the true value and its slope is not infinitely steep as it is supposed to be. The incident wave front does not reach the back apex of the shell at $\theta=\pi$; the pressure there is initiated at $T=0.81245$ by the shell response and later at $T=2.57079$ reinforced by the diffracted incident wave. Again, apart from small neighborhoods of 


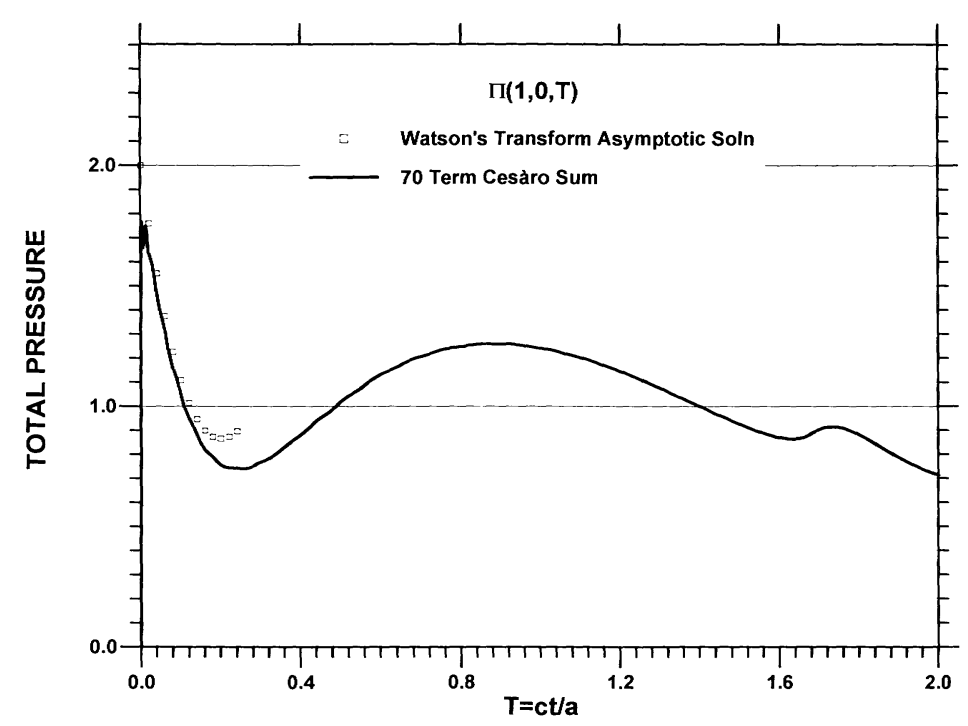

FIGURE 13 Comparison of the 70-term Cesàro sum representation of $\Pi(1,0, T)$ to the Watson's transformation solution.

the wave fronts, the pressure time histories in Fig. 14 have adequately converged. It is interesting to note that the values of the total pressure on the shell are very much above zero for all times. Therefore, for the case of a step incident wave, cavitation will not occur at the shell. It is also obvious that cavitation at the shell will occur for short incident waves and more likely occur near the back apex.

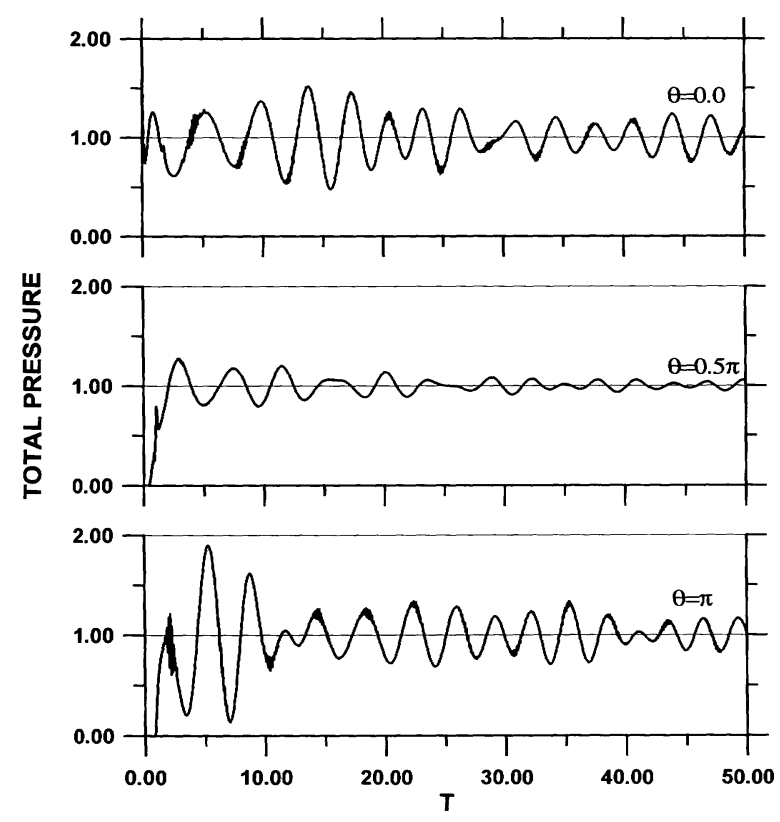

FIGURE 14 Time histories of $\Pi(1, \theta, T)$ for $0.0 \leq$ $T \leq 50.0$.

\section{SUMMARY}

Although a detailed solution to the transient interaction of plane acoustic waves with a spherical elastic shell was obtained more than a quarter of a century ago, two primary deficiencies existed, namely, meaningful solution histories of the higher time derivatives such as acceleration and pressure were not generated due to an insufficient number of terms in the series solution, and uniform convergence around the discontinuous step wave front was not obtained because of the Gibbs' phenomenon. These two deficiencies have been remedied with the advent and availability of more powerful computers and increased sophistication of computational algorithms. The utility of the classical modal series for this type of problem in particular, and for transient dynamic problems in general, is now greatly enhanced, because a similarly convergent solution is still difficult to obtain with more general solution techniques.

Many terms (up to 70) of the series solution are now accurately computed, and the $(\mathrm{C} 1)$ Cesàro summation of the series is employed to effectively eradicate all spurious oscillations due to the Gibbs' phenomenon. Except for small neighborhoods about the wave fronts, the series solution now converges uniformly toward the solution. The early time waveform of the velocity time history and the time of its maximum can be accurately defined, and meaningful solution histories for the higher time derivatives (e.g., shell acceler- 
ation and the pressure acting on the shell) are obtained. Refined numerical results are presented; these results are particularly useful for validation of computational models that attempt to reproduce the higher time derivatives, which are significantly more difficult to obtain.

The authors acknowledge the partial support of ONR 333 (Dr. Judah Goldwasser), under the ONR Undersea Warheads and Explosives Project, Modeling and Simulation of Warhead/Target Interactions Task. The authors also wish to express their appreciation to Professor Thomas L. Geers of the University of Colorado for his critical discussions.

\section{REFERENCES}

Berger, B., 1972, “Application of Cesàro Mean to the Transient Interaction of a Spherical Acoustic Wave and a Spherical Elastic Shell," Journal of Applied Mechanics, Vol. 39, pp. 623-626.

Carslaw, H. S., 1930, Introduction to the Theory of Fourier's Series, and Integrals, 3rd rev. ed., Dover Publications, Inc., Chap. IX.

Chan, K. K., and Atkatsh, 1991, “EPSA Topics: Transient Response of Submerged Spherical Shells,' in Proceedings of the 62nd Shock and Vibration Symposium, Vol. III, Springfield, VA, October 29-31, pp. 578-586.

Chan, S. K., 1990, “A Modified Finite Element Procedure for Underwater Shock Analysis," in Proceedings of the 61st Shock and Vibration Symposium, Vol. III, Pasadena, CA, October, 16-18, pp. 87-101.

Chan, S. K., 1992, “An Improvement in the Modified Finite Element Procedure for Underwater Shock Analysis," in Proceedings of the 63rd Shock and Vibration Symposium, Vol. II, Las Cruces, NM, October 27-29, pp. 616-621.

Chisum, J. E., and Shin, Y. S., 1995, “A Coupled Lagrangian-Eulerian and Multi-Material Eulerian Analysis in Underwater Shock Research," in Y. S. Shin, Structures Under Extreme Loading Conditions, PVP-Vol. 299, ASME, New York, pp. 33-38.

Geers, T. L., and Ju, T. H., 1994, “A Computer Program for a Canonical Problem in Underwater Shock," Shock And Vibration, Vol. 1, No. 4, pp. 331-337.

Hibbitt, Karlsson \& Sorensen, Inc., 1992, ABAQUSExample Problems Manual, Version 5.2, pp. 9.1.21-9.1.2-10.

Huang, H., 1969, "Transient Interaction of Plane Acoustic Waves with a Spherical Elastic Shell," Journal of the Acoustical Society of America, Vol. 45, pp. 661-670.
Huang, H., 1970, “An Exact Analysis of the Transient Interaction of Acoustic Plane Waves with a Cylindrical Elastic Shell," Journal of Applied Mechanics, Vol. 37, pp. 1091-1099.

Jenkins, M. A., 1975, "Zeros of a Real Polynomial," ACM Transactions in Math Software, Vol. 1, pp. 178-189.

Jones-Oliveira, J. B., and Harten, L. P., 1994, “Transient Fluid-Solid Interaction of Submerged Spherical Shells Revisited: Proliferation of Frequencies and Acoustic Radiation Effects,' Journal of the Acoustical Society of America, Vol. 96, pp. 918-925.

Milenkovic, V., and Raynor, S., 1966, "Reflection of a Plane Acoustic Step Wave from an Elastic Spherical Membrane," Journal of the Acoustical Society of America, Vol. 39, pp. 556-563.

Payton, R. G., 1960, "Transient Interaction of an Acoustic Wave with a Circular Cylindrical Elastic Shell," Journal of the Acoustical Society of America, Vol. 32, pp. 722-729.

Press, W. H., Flannery, B. P., Teulkosky, S. A., and Vettering, W. T., 1992, Numerical Recipes, 2nd ed., Cambridge University Press, New York, pp. 362-371.

Swegle, J. W., and Attaway, S. W., 1995, "On the Feasibility of Using Smoothed Particle Hydrodynamics for Underwater Explosion Calculations," Sandia National Laboratory Report, SAND95-0311, UC-705.

Tang, S. C., and Yen, D. H. Y., 1970, "Interaction of a Plane Acoustic Wave with an Elastic Spherical Shell,' Journal of the Acoustic Society of America, Vol. 47, pp. 1325-1333.

Waldo, G. V. Jr., 1994, "Approximate Model for the Pressure that Develops when an Acoustic Wave Interacts with a Curved and Compliant Surface,' in Proceedings of the 65th Shock \& Vibration Symposium, Vol. II, San Diego, October 31-November 3, pp. 185-198.

Whang, B., 1991, “DNA/NAVY Ship and Submarine Hardening Technology Committee UNDEX Code Validation Subcommittee Report,"' in Proceedings of the 62nd Shock and Vibration Symposium, Vol. III, Springfield, VA, October 29-31, pp. 627-637.

Whittaker, E. T., and Watson, G. N., 1958, A Course of Modern Analysis, 4th ed., Cambridge University Press, New York, p. 155.

Wright, J. P., 1991, “Direct Time Integration Methods for Structural Acoustics and Far Field Scattering Computations,"' ASME-AMD, Vol. 128, pp. 41-47; also accepted for publication in ASME Journal of Vibration and Acoustics, to appear.

Zhang, P., and Geers, T. L., 1993, "Excitation of a Fluid-Filled, Submerged Spherical Shell by a Transient Acoustic Wave," Journal of the Acoustical Society of America, Vol. 92, pp. 696-705. 

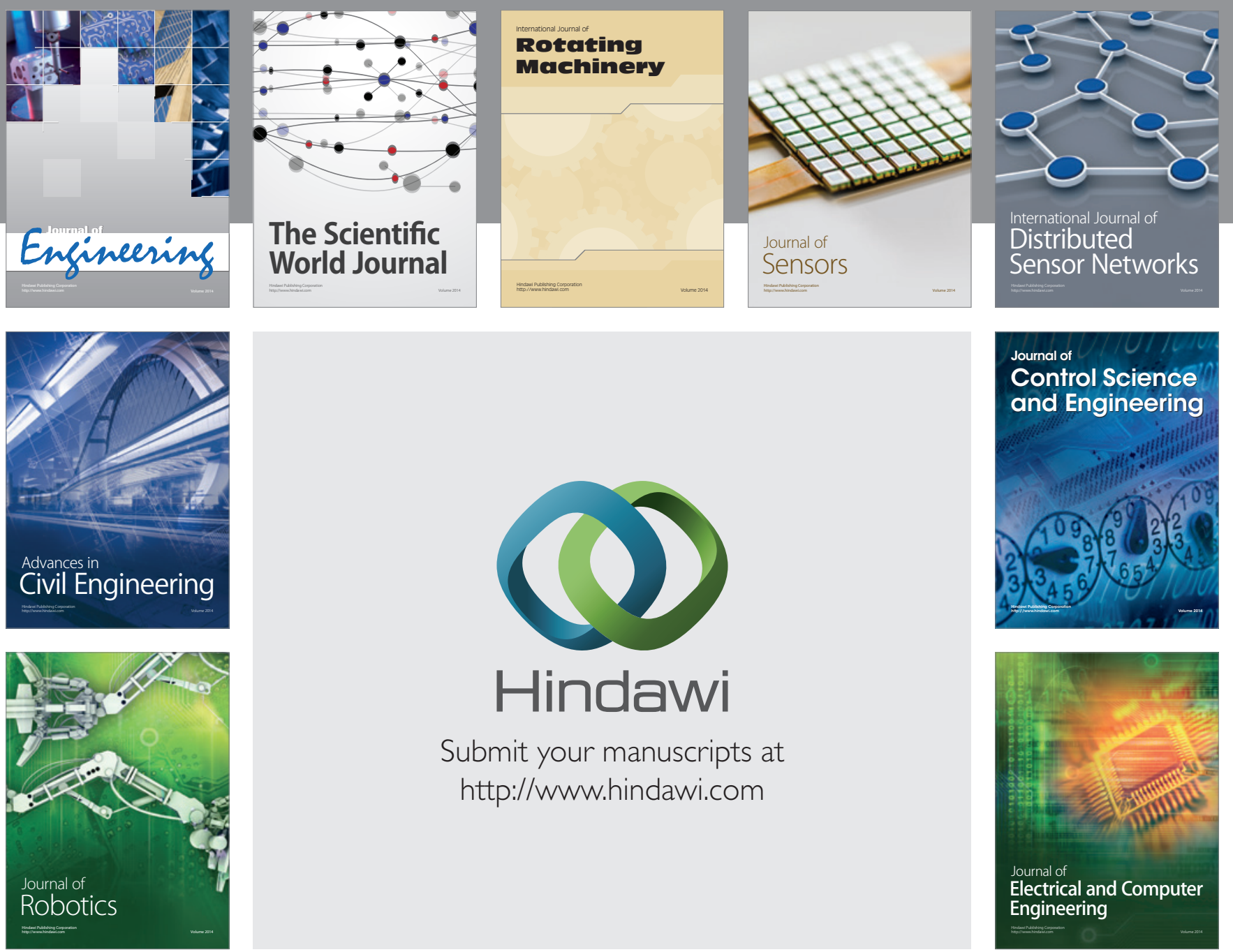

Submit your manuscripts at

http://www.hindawi.com
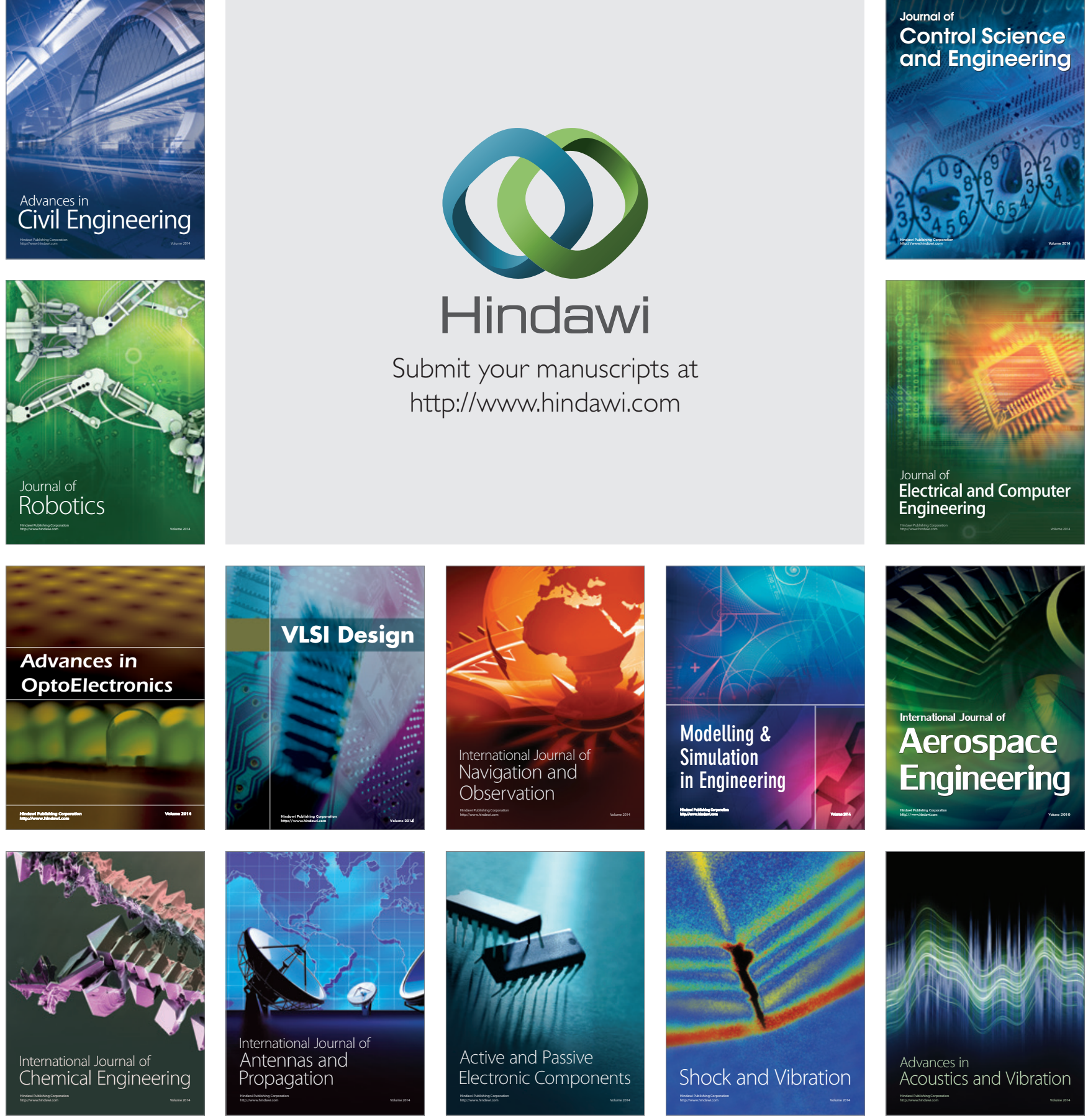